\title{
COMPARACIÓN DE LAS COMUNIDADES FITOPLANCTÓNICAS EN DOS EMBALSES DE RECIENTE CREACIÓN : RIAÑO Y VALPARAÍSO (ESPAÑA)
}

\author{
A. I. Negro ${ }^{1}$, C. de Hoyos ${ }^{1}$, A. del Rio ${ }^{2}$ y R. Le Cohu ${ }^{3}$ \\ 1. Area de Ecología. Facultad de Biología. Universidad de Salamanca. 37071 SALAMANCA (España) \\ 2. Area de Ecología. Facultad de Biología. Universidad de León. 24071 LEÓN ( España). \\ 3. Laboratoire d'Hidrobiologie. Université Paul Sabatier. 118, route de Narbonne. 31062 TOULOUSE (Francia) \\ PALABRAS CLAVE: Fitoplancton, variación estacional, embalse, sustrato, gestión.
}

\begin{abstract}
COMPARISON OF PHYTOPLANKTON COMMUNITIES IN TWO RECENTLY CONSTRUCTED RESERVOIRS: RIAÑO AND VALPARAISO (SPAIN)

Phytoplankton was studied in two recently constructed reservoirs situated in North-West Spain. Both belong to the same catchment area, but they are on different bedrock. Their comparison shows seasonal differences in phytoplakton abundance and composition. This seems to be more a reflection of the basin bedrock than of the young type of the ecosystems. The results lead us to suggest that good reservoirs management requires more studies on their phytoplankton.
\end{abstract}

\section{INTRODUCCIÓN}

Tradicionalmente los trabajos dirigidos a la gestión de embalses se han centrado en una caracterización físicoquímica del agua, pero además de ello es necesario abordar el estudio de la dinámica estacional del fitoplancton (LE COHU et al.,1991).

España ha pasado de ser un país casi sin lagos̄ a contar con unos mil embalses en menos de cincuenta años. Además se preve la construcción de un número elevado de ellos según se desprende del proyecto del nuevo Plan Hidrológico Nacional (pendiente de aprobación). A pesar de existir tal cantidad de embalses a lo largo de toda la red hidrológica, es de lamentar la escasez de trabajos en los que se haya realizado un seguimiento de las primeras etapas de llenado. Podemos citar el estudio del embalse de Sau (VIDAL, 1969, 1976).

El funcionamiento de un embalse está condicionado por el clima, características de la cuenca (tipo de sustrato, suelo y vegetación) y la actividad humana desarrollada en la misma. Este tipo de ecosistemas, sujetos normalmente a un importante aporte de nutrientes, regulan de diferente manera la entrada de éstos en función de la concentración en sales disueltas de sus aguas, que a su vez depende del sustrato geológico de la cuenca. Todo esto va a condicio- nar la biomasa y composición del fitoplancton. Debido a que en la Península Iberica se diferencian dos zonas distintas litológicamente (silícea y calcárea), los embalses españoles pueden dividirse en dos grupos según la composición iónica de sus aguas (MARGALEF et al.,1977; RIERA et al., 1990).

Nuestro trabajo se centra en la comparación de la flora algal de dos embalses de reciente creación, Riaño y Valparaíso, enclavados en la misma cuenca hidrográfica pero con sustrato diferente. Ya existen algunos estudios de la caracterización físico-química de sus primeras etapas (DEL RIO et al.,1990 ; BENGOECHEA, 1991).

\section{MATERIALES Y MÉTODOS}

Los muestreos se realizaron 2 años después del llenado de los embalses en una estación localizada, en ambos casos, a $200 \mathrm{~m}$ de la presa. Las profundidades máximas alcanzadas en ese punto durante la época de estudio fueron de 50 m en Valparaíso y de 60 m en Riaño.

Las muestras se tomaron en el epilimnion a lo largo de un ciclo estacional (mayo, agosto, octubre y febrero) en profundidades de 2,5 y $10 \mathrm{~m}$ durante la estratificación, y a 2 y $10 \mathrm{~m}$ en la época de mezcla.

Las muestras fueron recogidas con una botella hidrográ- 
fica tipo Van Dorn y las destinadas al recuento de fitoplancton se fijaron in situ con lugol. Los recuentos de las especies del fitoplancton se llevaron acabo según la metodología de Utermohl (LUND et al., 1958).

Los datos sobre química que se mencionan en este trabajo proceden de estudios paralelos que se realizaron sobre Riaño y Valparaíso y que aún no han sido publicados.

Las medidas de $\mathrm{pH}$, conductividad, oxígeno disuelto y temperatura se efectuaron in situ utilizando $\mathrm{pH}$-metro WTW, conductímetro WTW LF-91 y oxímetro WTW OXI-191. La clorofila se extrajo con acetona, previa filtración de las muestras, y se midió espectrofotométricamente. Al mismo tiempo el agua filtrada se empleó para la determinación de nutrientes. Para los fosfatos se empleó el método del ácido de cadmio y la cantidad de nitritos se calculó utilizando el método de la sulfanilamida. El amonio y la sílice se determinaron sobre muestras no filtradas para evitar contaminaciones de las muestras. En el caso del amonio a través del método del azul de indofenol y en el de la sílice se utilizó molibdato amónico, añadiendo ácido oxálico para evitar la interferencia del fosfato. La descripción detallada de estos métodos se encuentra en Standard Methods (APHA, 1985).

\section{ÁREA DE ESTUDIO}

Los embalses de Riaño y Valparaíso se hallan localizados ambos en la cuenca del río Duero (noroeste de España) y fueron creados para su explotación hidroeléctrica y para la reserva de agua de cara a la irrigación agrícola. Como podemos observar en la tabla 1, Riaño tiene una capacidad considerablemente mayor en comparación con Valparaíso, y cubre una superficie de casi el doble de hectáreas.

Valparaíso, situado a $850 \mathrm{~m}$. de altitud, se alimenta con las aguas del río Tera; está precedido por el embalse de Cernadilla. Riaño es un embalse de cabecera, situado a $1.100 \mathrm{~m}$ de altitud, en el curso alto del río Esla. Tanto Riaño como Valparaíso se encuentran en zonas donde se registran unas diferencias de temperatura atmosférica bastante acusadas. En Valparaíso la temperatura media anual es algo más elevada y la pluviosidad ligeramente más baja (tabla 2).

Otra de las características diferenciales de estos dos embalses es que a pesar de su relativa proximidad geográfica, sus cuencas se asientan sobre litologías bien diferentes. Riaño está situado en una zona calcárea, mientras que Valparaíso se encuentra en zona silícea (fig.1). Las dife-
TABLA 1.- Parámetros morfométricos TABLE 1.- Morphometnc parameters.

\begin{tabular}{lcc}
\hline & Riaño & Valparaíso \\
\hline Volumen $\left(\mathrm{Hm}^{3}\right)$ & 651 & 168,5 \\
Superficie $(\mathrm{Ha})$ & 2230 & 1223 \\
Altitud $(\mathrm{m})$ & 1100 & 850 \\
Altura presa $(\mathrm{m})$ & 102.5 & 67 \\
\hline
\end{tabular}

TABLA 2.- Parámetros climatológicos.

TABLE 2.- Climatic parameters.

\begin{tabular}{lcc}
\hline & Riaño & Valparaíso \\
\hline Temperatura media anual $\left({ }^{\circ} \mathrm{C}\right)$ & 8,5 & 11 \\
Precipitación media anual $(\mathrm{mm})$ & 1300 & 800 \\
\hline
\end{tabular}

rentes características geológicas de sus cubetas y cuencas de drenaje hacen que las características químicas del agua de Riaño y Valparaíso sean también diferentes. El agua de Valparaíso tiene una baja concentración de sales, pH relativamente bajo y también unos niveles bajos de alcalinidad, derivado todo ello de la escasa solubilidad de los minerales que forman la roca del sustrato (gneises cámbricos). Por el contrario, en Riaño encontramos una mayor concentración de sales y mayor alcalinidad en el agua, como ocurre en todos los embalses situados sobre

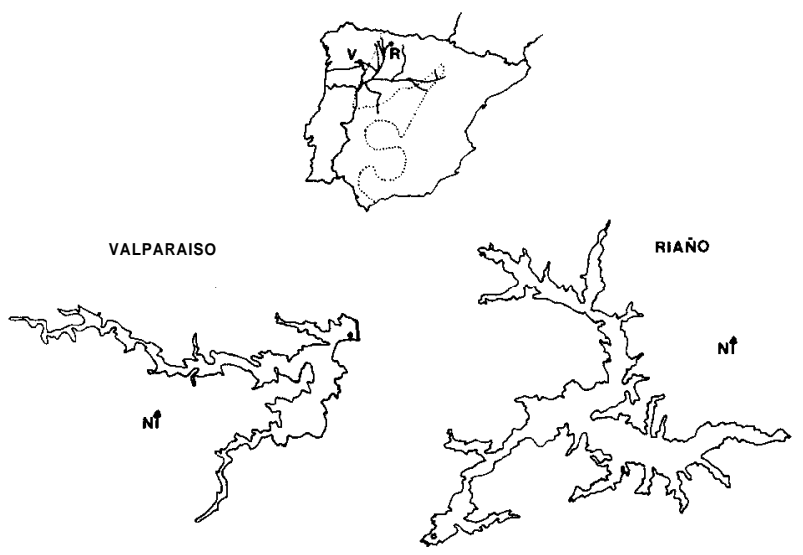

FIGURA 1.- Localización gegráfica de Riaño y Valparaíso en la Península Ibérica.

FIGURE 1.- Geographic location of Riaño and Valparaíso in the Iberian Península.

sustrato calcáreo (tabla 3).

Estos dos embalses iniciaron su llenado en 1988, pasando por un período inicial de anoxia en el hipolimnion (que aún se detectó en Valparaíso cuando se realizó este estudio). El agua ha experimentado oscilaciones de nivel dife- 
TABLA 3.- Rangos de variación de algunos parámetros químicos TABLE 3.- Range of some chemical parameters.

\begin{tabular}{|c|c|c|c|c|c|c|c|}
\hline & $\begin{array}{c}\text { Conductividad } \\
\qquad(\mu \mathrm{S} / \mathrm{cm})\end{array}$ & $p H$ & $\begin{array}{c}\text { Alcalinidad } \\
\text { (meq I) }\end{array}$ & $\begin{array}{l}\text { Calcio } \\
(\mathrm{mg} / 1)\end{array}$ & $\begin{array}{c}\text { Magnesio } \\
(\mathrm{mg} / \mathrm{l})\end{array}$ & $\begin{array}{l}\text { Sodio } \\
(\mathrm{mg} / \mathrm{l})\end{array}$ & $\begin{array}{l}\text { Potasio } \\
\text { (mg'l) }\end{array}$ \\
\hline Valoaraiso & 22.30 & $59-71$ & $0.13 \cdot 0.19$ & $0.5-1.6$ & $0.2-0.4$ & $0.7 \cdot 1.2$ & 0.20 .5 \\
\hline Riaño & 100130 & $75-86$ & $0.5 \cdot 1.77$ & $15.87 \cdot 23.27$ & $0.5 .8 \quad 1$ & $1.13 \cdot 2.05$ & $0.6-3.7$ \\
\hline
\end{tabular}

rentes. En Valparaíso prácticamente no varía el nivel del agua, pues el embalse de Cernadilla se utiliza para regularlo. Riaño sin embargo ha sufrido oscilaciones de nivel bastante fuertes durante los dos primeros años de vida, aunque durante el período estudiado éste se mantuvo estable.

Son embalses monomícticos. Durante el período de estudio Riaño inicia la estratificación ligeramente antes que Valparaíso (Abril). La termoclina se rompe en los dos casos a finales de Septiembre (fig. 2). También se producen durante este mes los máximos en la transparencia del agua (Disco de Secchi de $4 \mathrm{~m}$. en Valparaíso y de $8.5 \mathrm{~m}$. en Riaño).

La concentración de nutrientes en ambos embalses es
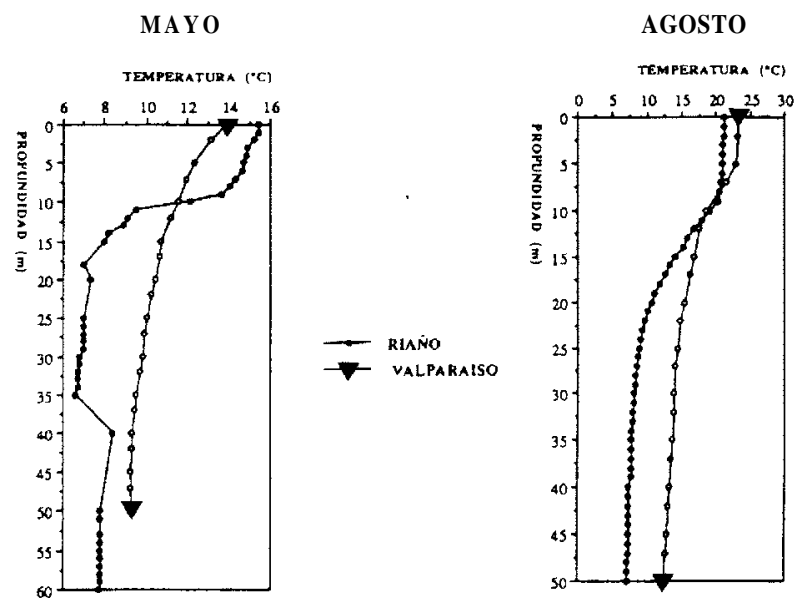

FIGURA 2.- Perfiles térmicos en dos momentos de la estratificación FIGURE 2.- Thermic profiles during stratification.

TABLA 4.- Intervalos de las concentraciones de los principales nutrientes en el epilimnion

TABLE 4.- Range of main nutrients concentration in the epilimnion.

\begin{tabular}{lccc}
\cline { 2 - 4 } & $\mathrm{N}$ & $\mathrm{P}$ & $\mathrm{Si}$ \\
& $(\mu \mathrm{N}$ inorg./1) & $($ yg P-PO $/ 1)$ & $(\mathrm{mg} \mathrm{Si} / 1)$ \\
\hline \hline Valparaíso & $3.2-26$ & $0.3-3.0$ & $0-0.7$ \\
Riaño & $20-250$ & $0-4.5$ & $0-2.12$ \\
\hline
\end{tabular}

baja. Destaca la escasez de $\mathrm{N}$ inorgánico en el embalse de Valparaíso (tabla 4).

\section{RESULTADOS}

\section{Composición y abundancia de las comunidades fitoplanctónicas.}

La composición del fitoplancton en estos dos embalses es notablemente diferente (fig. 3). En Riaño se observan máximos de clorofila a $(7 \mu \mathrm{g} / \mathrm{l})$ y $\mathrm{n}^{\mathrm{O}}$ de células por mililitro (11.850) en primavera y verano, máximos caracterizados por la presencia de diatomeas. En invierno el número de células es muy bajo ( 165 cels/ml). Esta es la sucesión estacional de fitoplancton típica descrita para lagos templados (MARGALEF, 1983; REYNOLDS, 1984; SOMMER, 1986).

Por el contrario la evolución anual de la comunidad fitoplanctónica en Valparaíso fue bastante atípica durante el período de estudio. Los máximos de fitoplancton corresponden al invierno (17 yg clorofila $a / 1 ; 72.219 \mathrm{cels} / \mathrm{ml}$ ) . Este fenómeno se ha constatado durante los primeros años de vida de algunos embalses (VIDAL, 1969). Este máximo invernal está constituido por algas cianofíceas, clorofíceas y diatomeas. Hay que destacar también en Valparaíso la gran cantidad de cianofíceas durante el verano y otoño. El masivo desarrollo de estas algas hace que el mayor número de células por mililitro se alcance en octubre (148.157 cels/ml). Esto no se corresponde con el valor máximo de biomasa debido al pequeño tamaño de estas células.

\section{Dinámica estaciona1 de los grupos taxonómicos más representativos}

\section{CIANOFÍCEAS}

Valparaíso supera desmesuradamente a Riaño en cuanto a la cantidad de cianofíceas (fig. 4). Es un grupo de algas que se halla presente en Valparaíso durante todo el ciclo anual, con un máximo al principio del otoño (142.333 cels/ml), coincidiendo con el agotamiento de los nutrientes tras la época estival. Posteriormente desciende el nivel a lo largo de los meses siguientes, pero curiosamente se mantiene como un grupo importante dentro del fitoplancton invernal $(74.567 \mathrm{cels} / \mathrm{ml})$.

La especie mayoritaria es Aphanothece clathrata. Durante el verano y otoño se desarrollan además especies con heterocistes (Anabaena sp.), aunque con una importancia 

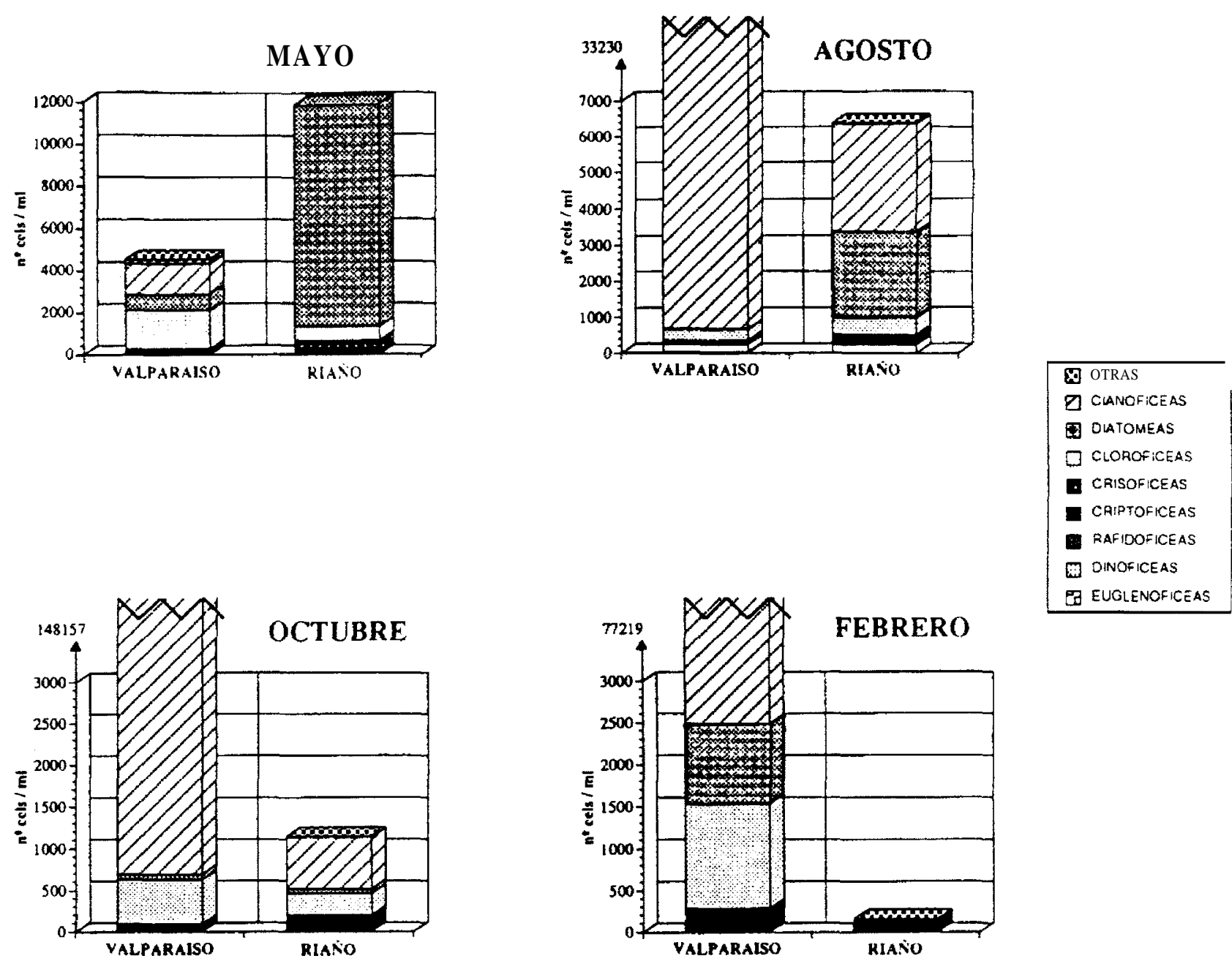

FIGURA 3.- Variación estacionalen la composición y abundancia de las comunidades fitoplanctónicas FIGURE 3.- Seasonal changes in the composition and abundance of phytoplankton communities.

menor que A. clathrata. En Riaño, la aparición de cianofíceas en verano y otoño es puntual $(2.963 \mathrm{cels} / \mathrm{ml})$, y no contribuye de manera importante en la biomasa del fitoplancton. En ningún caso son especies con heterocistes.

Las aguas ricas en calcio regulan el estado trófico del ecosistema debido a la eliminación del fósforo del epilimnion al precipitar con el ión calcio. Los ecosistemas acuáticos asentados en sustrato silíceo carecen de este sistema de regulación; la escasez de calcio en el agua les hace más sensibles al aporte de nutrientes (RIERA, et al., 1990). Esta situación condiciona una relación entre los nutrientes principales que favorece el desarrollo de cianofíceas. En un extenso estudio relizado en 1976 sobre em- balses españoles se establecieron unos valores de cociente N/P característicos de la zona silícea y calcárea de 20 y 100 respectivamente (MARGALEF et al., 1977). Riaño y Valparaíso presentan unas relaciones N/P que siguen esa norma. Valparaíso es bastante pobre en nitrógeno y sus índices N/P resultan bastante bajos. En Riaño sin embargo el fósforo es indetectable en muchas ocasiones, pero muestra concentraciones de nitrógeno bastante elevadas, disparándose el valor del cociente N/P (tabla 4). Todo esto parece estar bien relacionado con la práctica ausencia de cianofíceas en Riaño y su extraordinaria abundancia en Valparaíso.

Tradicionalmente se ha considerado que las temperatu- 


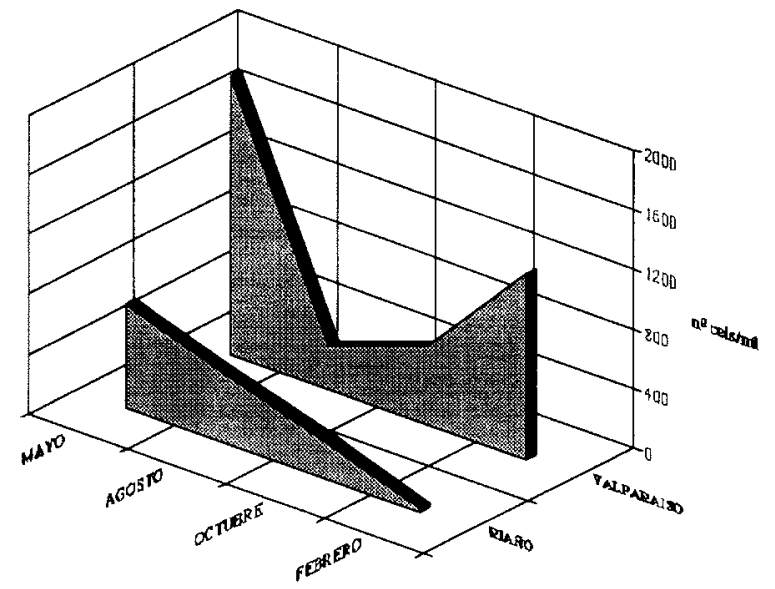

FIGURA 4.- Dinámica estacional de las cianofíceas.

FIGURE 4.- Blue-green algae seasonal dynamics.

ras altas favorecen el crecimiento de las cianofíceas (HAMMER, 1964; REYNOLDS et al.,1975). En la bibliografía existen numerosas referencias acerca de los rangos de temperatura óptimos a los cuales se desarrollan diversas especies de estas algas (REYNOLDS, 1971; FOGG et al., 1973; TILMAN et al., 1986). El ciclo que siguen las cianofíceas en Valparaíso no concuerda con los resultados obtenidos por estos autores (fig. 4).

\section{DIATOMEAS}

Son el grupo de algas dominante en Riaño.

Aunque Valparaíso se asienta sobre un sustrato de naturaleza silícea, la concentración de sílice en el agua es menor que en Riaño (tabla 4), donde la concentración de este elemento es más favorable para el desarrollo de las diatomeas. El máximo crecimiento de la población de diatomeas se produce en Riaño durante la primavera ( 10.510 cels $/ \mathrm{ml})$ pero, cuando se establece la termoclina, la cantidad de estas algas disminuye a un ritmo bastante rápido. Esto se debe a que la gran masa de diatomeas primaverales acaba totalmente con la reserva silícea del agua en la capa trofogénica, impidiendo así el mantenimiento de la población (LUND, 1965; MARGALEF, 1983; SOMMER, 1983; BAILEY-WATTS, 1976). Durante la primavera la especie que predomina en Riaño es Asterionella formosa y en verano Fragilaria crotonensis. A. formosa puede desarrollarse perfectamente con escasas concentraciones de fósforo, como las de Riaño, siempre que la sílice sea abundante (MARGALEF, 1983). La concentración de sílice necesaria para el crecimiento de este alga no debe ser inferior a $0,2 \mathrm{mg} \mathrm{Si} / \mathbf{l}$ (LUND,

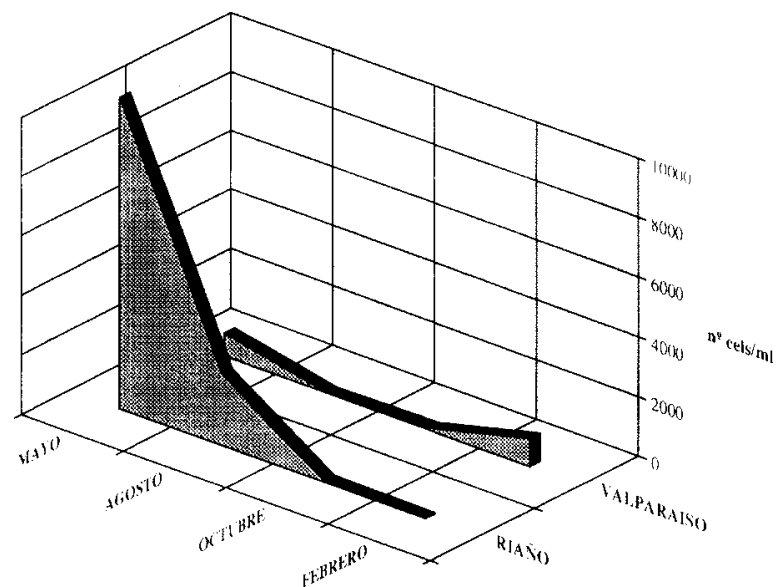

FIGURA 5.- Dinámica estaciona1 de las diatomeas.

FIGURE 5.- Diatoms seasonal dynamics.

1950). En general las diatomeas son buenos competidores por el fósforo, y sustituyen a otros grupos de algas cuando los cocientes N/P y Si/P son elevados y las temperaturas bajas (TILMAN et al., 1986; LøVSTAD \& BJøRNDALEN, 1990). Estas condiciones se observan en Riaño.

En Valparaíso, al contrario que en Riaño se obseva un máximo invernal $(955 \mathrm{cels} / \mathrm{ml})$ al que contribuye principalmente Tabellaria fenestrata. También se encuentra Asterionella formosa, aunque en cantidades inferiores a las de Riaño.

\section{CLOROFÍCEAS}

En ambos embalses el máximo de clorofíceas se produce durante la primavera ( 690 cels/ml en Riaño y 1.865 cels/ml en Valparaíso), y desciende a lo largo del verano. Pero mientras en Valparaíso el descenso es brusco y a principios del otoño vuelve a incrementarse el número de clorofíceas, en Riaño la tendencia iniciada en verano se mantiene, hasta que prácticamente desaparecen en invierno. Contribuyen a estas diferencias algunas de las especies de desmidiáceas presentes en Valparaíso que apenas se encuentran en Riaño, las cuales experimentan, a partir del otoño, un aumento cuantitativo y cualitativo (destaca el número de células del género Staurastrum sp.). La relativa acidez del agua y la baja concentración salina de Valparaíso favorecen a estas algas, que por el contrario escasean en aguas ricas en calcio como las de Riaño (MARGALEF, 1983).

En los dos embalses podemos encontrar principalmente algas de los órdenes Chlorococcales y Tetrasporales, 


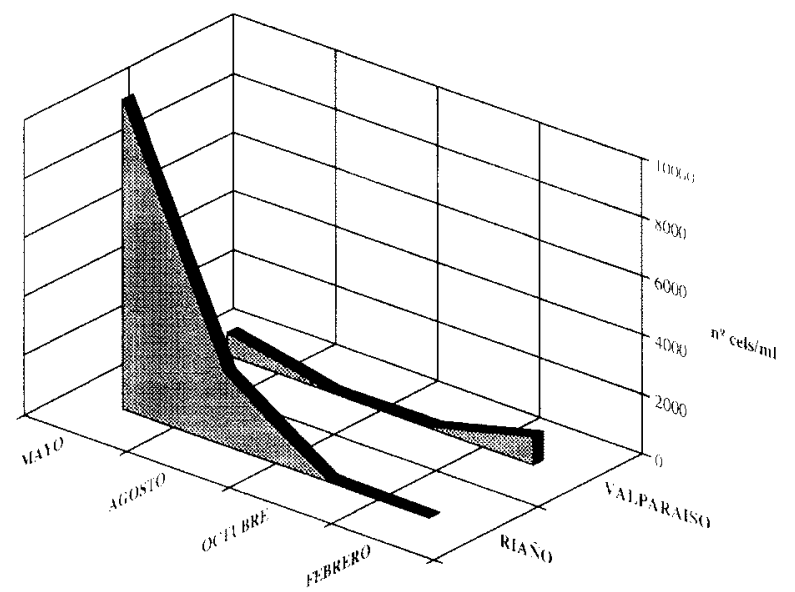

FIGURA 6.- Dinámica estacional de las cloroficeas FIGURE 6.- Green algae seasonal dynamics.

sin que se observe una dominancia clara de alguna especie.

\section{OTROS GRUPOS}

Las Crisofíceas y Criptofíceas también están representadas en Valparaíso y en Riaño (Dinobryon sp., Rhodomonas sp., Cryptomonas sp.), pero el número de especies y abundancia es menor. Los máximos valores registrados en las crisofíceas fueron para Valparaíso y Riaño de 131 y 626 cels/ml respectivamente. En criptofíceas 212 y 221 cels $/ \mathrm{ml}$ respectivamente.

Cabe mencionar la presencia de rafidofíceas en Valparaíso, con una sola especie perteneciente al género Gonyostomum sp., y que cuantitativamente es relevante durante el verano y otoño $(14 \mathrm{cels} / \mathrm{ml})$ contribuyendo además en buena parte a la biomasa total del fitoplancton por su tamaño celular. Ha sido relacionado con turberas y la presencia de ácidos húmicos en el agua (ROSEN, 1981; BOURRELLY, 1985). La existencia de un extenso sistema de turberas aguas arriba del embalse de Valparaíso puede explicar la aparición de esta especie. Durante los últimos años se ha concedido un especial interés a su estudio, debido a los desarrollos masivos de este alga encontrados en algunos embalses como el de Pareloup en Francia (LE COHU et al., 1989), y por los problemas que causa su presencia, en grandes cantidades, a los bañistas (reacciones alérgicas e irritaciones dérmicas) (BJöRNDALEN \& LöVSTAD, 1984; MANNINEN \& KIVINEN, 1985).

Euglenofíceas y xantofíceas tienen escasa representación en ambos embalses.

\section{CONCLUSIONES}

La composición y evolución anual de la comunidad fitoplanctónica de estos dos embalses son marcadamente diferentes. El ciclo estacional de Valparaíso puede calificarse de atípico por localizarse el máximo anual en invierno, mientras que Riaño sigue un patrón similar a lo encontrado en lagos templados europeos, con máximos en primavera y verano. La producción en ambos embalses es baja, siendo la de Riaño inferior a la de Valparaíso.

Parece que dos años después de su llenado las comunidades de fitoplancton establecidas en estos embalses son más un reflejo de las características de la cuenca que del carácter joven del ecosistema. La presencia mayoritaria de cianofíceas en Valparaíso y de diatomeas en Riaño guarda una estrecha relación con la concentración de nutrientes principales, que se deriva del tipo de sustrato, silíceo y calcáreo respectivamente, en el que se asientan estos embalses.

Asímismo es destacable la abundancia del género Gonyostomum sp. en Valparaíso, que se ha revelado como alga "invasora" en embalses europeos. La concurrencia de Gonyostomum sp. junto con cianofíceas en este embalse, debe ser motivo de un cuidado especial del agua, por el carácter tóxico de ambos tipos de algas.

Consideramos por todo esto interesante tener en cuenta en la gestión de embalses, no sólo parámetros físico-químicos, sino el estudio de las poblaciones algales.

\section{BIBLIOGRAFÍA}

APHA, 1985. Standard methods for the examination of water and wastewater. APHA. AWWH.WPCF

BAILEY-WATTS, A.E., 1976. Planktonic diatoms and some diatom-silica relations in a shallow eutrophic Scottish loch. Freswater Biology, 6: 69-80.

BENGOECHEA, C., 1991. Valparaiso dam - Tera river first filling experiences. International Commission on Large Dams, 64(5): 67-83.

BJöRNDALEN, K. \& O. LöVSDTAD, 1984. En regionalumdersoklse av innsjoer $\mathbf{i}$ ostfold. Eutrafier ing og problemealger van., 1: 123-132.

BOURRELLY, P. 1985. Les algues d'eau douce. III. Editions N. Boubée et Cie. Paris : 606 pp.

FOGG, G.E., STEWART W.D., FAY, P. \& A.E. WALSBY, 1973. The Blue-green Algae. Academic Press. London and New York: $450 \mathrm{pp}$.

LE COHU, R., GUITARD, J., COMOY, N. \& J. BRA- 
BET, 1989. Gonyostomurn semen (Raphidophycées), nuisance potentielle des grands réservoirs français? L'exemple du lac de Pareloup. Arch. Hydrobiol., 117(2): 225-236.

LE COHU R., COMOY N. GUITARD J. \& J. BRABET, 1991. Périodicité du phytoplancton dans un réservoir de moyenne profondeur: le lac de Pareloup (Massif central, France), un exemple de succession cyclique. Annls. Limnol., 27(3): 197-214.

LöVSTAD, O. \& K. BJöRNDALEN, 1990. Nutrients $(\mathrm{P}, \mathrm{N}, \mathrm{Si})$ and growth conditions for diatoms and Oscillatoria spp. in lakes of south-eastern Norway. Hydrobiologia, 196: 255-263

LUND, J.W.G., KIPLING C. \& E.D. LE CREN E.D., 1958. The inverted microscope of estimation algal numbers and the statistical basis of estimations by counting. Hydrobiologia, 2: 143-170.

LUND, J.W.G., 1965. The ecology of the freshwater phytoplankton. Biol. Rev., 40: 231-293.

MANNINEN, P. \& J. KIVINEN, 1985. Gonyostomum semen (EHR.)DIES. (Chloromonadophyceae)-Levan esüntymisesta ja veden laadusta erailla vesistoalueilla. Tiedatus 266. Vattenstyrelsen, Helzingfors: 44 pp.

MARGALEF, R., PLANAS, D., ARMENGOL, J., VIDAL, A., PRAT, N., GUISET, A., TOJA, J. \& M. ESTRADA, 1977. Lirnnología de los embalses españoles. Dirección General de Obras Hidráulicas. Ministerio de Obras Públicas. Madrid: 422 pp.

MARGALEF, R., 1983. Lirnnología. Ediciones Omega. Barcelona: $1010 \mathrm{pp}$.

REYNOLDS, C.S., 1971. The ecology of the planktonic blue-green algae in the North Shropshire meres. Field Studies, 3: 409-432.

REYNOLDS, C.S., 1975. Water-Blooms. Biol. Rev., 50: 437-481.
REYNOLDS, C.S., 1984. The ecology of freshwater phytoplankton. Cambridge University Press. Cambridge: 384 pp.

RIERA, J.L. \& J.A. MORGUí, 1990. Limnología regional de los embalses españoles. Mundo Cientlfico, 10 (104): 720-726.

RíO, DEL A., GRAUPERA, E., FERNANDEZ, C. \& E. LUIS, 1991. Physical-chemical research of a mountain reservoir in filling phase. Verh. Internat. Verein. Limnol., 24: 1396-1400.

ROSEN, G., 1981. Phytoplankton indicators and their relations to certain chemical and physical factors. Limnologica, 13(2): 263-290.

SOMMER, U. \& H.H. STABEL, 1983. Silicon cosumption and population density changes of dominant planktonic diatoms in lake Constance. Journal of Ecology, 71: 119-130.

SOMMER, U., GLIWICZ, Z.M., LAMPERT, W. \& A. DUNCAN, 1986. The P.E.G. model of seasonal succession of planktonic events in freshwaters. Archiv. Hydrobiol., 106(4): 433-471.

TILMAN, D., KIESLING, R., STERNER, R., KILHAM, S. \& F. A. JOHNSON, 1986. Green, bluegreen and diatom algae: Taxonomic differences in competitive ability for phosphorus, silicon and nitrogen. Arch. Hydrobiol., 106 (4): 473-485.

VIDAL, A., 1969. Evolution d'un lac de barrage dans le NE. de 1'Espagne pendant les quatre premières années de service. Verh. Internat. Verein. Limnol., 17: 191-200.

VIDAL, A., 1976. Eutrofización del embalse de Sau en el transcurso de sus primeros años (1963-1972). II Asamblea Nacional de Geodesia y Geofísica. Servicio de Explotación Confederación Hidrográfica del Pirineo Oriental. Barcelona: 1955-1997. 